\title{
Higher-order spacing ratios in random matrix theory and complex quantum systems
}

\author{
S. Harshini Tekur * Udaysinh T. Bhosale and M. S. Santhanan \\ Indian Institute of Science Education and Research, Dr. Homi Bhabha Road, Pune 411 008, India
}

\begin{abstract}
The distribution of the ratios of nearest neighbor level spacings has become a popular indicator of spectral fluctuations in complex quantum systems such as the localized and thermal phases of interacting many-body systems, quantum chaotic systems, and in atomic and nuclear physics. In contrast to the level spacing distribution, which requires the cumbersome and at times ambiguous unfolding procedure, the ratios of spacings do not require unfolding and are easier to compute. In this work, for the class of Wigner-Dyson random matrices with nearest neighbor spacing ratios $r$ distributed as $P_{\beta}(r)$ for the three ensembles indexed by $\beta=1,2,4$, their $k$-th order spacing ratio distributions are shown to be identical to $P_{\beta^{\prime}}(r)$, where $\beta^{\prime}$, an integer, is a function of $\beta$ and $k$. This result is shown for Gaussian and circular ensembles of random matrix theory and for several physical systems such as spin chains, chaotic billiards, Floquet systems and measured nuclear resonances.
\end{abstract}

\section{INTRODUCTION}

Spectral fluctuations in complex quantum systems contain information about their physical character. In tight binding models and crystalline lattices that display metal to insulator transition, the metallic and insulator regimes are distinguishable based on their spectral fluctuations 1 . In interacting spin chains, many body localized and thermal phases also carry distinct spectral signatures ${ }^{2}$. In quantum systems with chaotic classical limit ${ }^{3}$, the fluctuations indicate if the system is integrable, chaotic or a mixture of both these types of dynamics 4 .

Of the spectral fluctuation measures modeled through random matrix theory $(\mathrm{RMT})^{\frac{5}{4}}$, the most popular one is the nearest neighbor level spacings, $s_{i}=E_{i+1}-E_{i}$, where $E_{i}, i=1,2, \ldots$ are the discrete eigenvalues of a quantum operator. For time-reversal invariant systems without spin degree of freedom, RMT predicts that the spacings are Wigner distributed ${ }^{6}, P(s)=(\pi / 2) s \exp \left(-\pi s^{2} / 4\right)$ indicating the presence of level repulsion. In physical systems, Wigner distribution is associated with the metallic regime of tight binding models $\frac{7}{}$, thermal phase of many-body systems ${ }^{8}$, chaotic quantum systems $\frac{9}{\underline{ }}$ such as coupled oscillators 10 and atoms in strong external fields 11 and many others ${ }^{12}$. This is in contrast to the class of integrable systems such as many body localized phase of interacting systems 13 , which display level clustering through Poissonian spacing distribution, $P(s)=$ $\exp (-s)$.

In practice, spacing distributions have to be computed after removing the system dependent spectral features, i.e., the average part of the density of states, through a cumbersome and non-unique numerical procedure of unfolding the spectra. Further, in many-body systems (for example, Bose-Hubbard model) at large interaction strengths, the density of states is not a smooth function of energy and hence separating it into an average and fluctuating part, a pre-requisite for spectral unfolding, becomes non-trivial ${ }^{14,15}$.

These difficulties have been overcome through the use of spacing ratio $14 r_{i}=s_{i+1} / s_{i}, i=1,2, \ldots$, since it does not depend on the local density of states and con- sequently does not require unfolding. The RMT averages for the spacing ratios, drawn from three standard random matrix ensembles with co-dimension $\beta=1,2$ and 4 corresponding to the Gaussian orthogonal ensemble (GOE), unitary ensemble (GUE) and symplectic ensemble (GSE), have been obtained as ${ }^{16,17}$,

$$
P(r, \beta)=C_{\beta} \frac{\left(r+r^{2}\right)^{\beta}}{\left(1+r+r^{2}\right)^{1+\frac{3}{2} \beta}},
$$

where $C_{\beta}$ is a constant that depends on $\beta$.

Several variants of spacing ratios have been studied recently in different contexts ${ }^{18}$. However, few results exist for the distribution of non-overlapping higher-order spacing ratio $\frac{19}{2}$ defined by

$$
r_{i}^{(k)}=\frac{s_{i+k}^{(k)}}{s_{i}^{(k)}}=\frac{E_{i+2 k}-E_{i+k}}{E_{i+k}-E_{i}}, \quad i, k=1,2,3, \ldots
$$

Nearest neighbor spacing ratio $r$ probes fluctuations in spectral scales of the order of unit mean spacing, whereas $r^{(k)}$ probes fluctuations in spectral interval of $k$ mean spacings. In many physical situations, knowledge of spectral fluctuations at larger spectral intervals is useful. For quantum chaotic systems with a classical limit, semiclassical theories ${ }^{20}$ dictate that the higher-order spectral fluctuations would be related to short time periodic orbits, effectively acting as a probe of short time dynamics 9 , at shorter than Heisenberg time-scale. The rare-region effects or Griffith effects 21 in the vicinity of many-body localization transition influences the transport and entanglement properties, whose time-scales can be probed by the higher-order spacing ratios.

\section{DISTRIBUTION OF HIGHER-ORDER SPACING RATIOS}

Thus, higher-order spacing ratios have profound physical implications apart from being an intrinsic object of interest in random matrix theory $22-24$. In this work, for the random matrices, of order $N>>1$, belonging to the 
Gaussian and circular ensembles of random matrix theory, we present compelling numerical evidence to demonstrate an elegant relation between non-overlapping $k$-th order spacing ratio distribution $P^{k}(r, \beta)$ and the nearest neighbor spacing ratio distribution $P\left(r, \beta^{\prime}\right)$ :

$$
\begin{aligned}
P^{k}(r, \beta) & =P\left(r, \beta^{\prime}\right), \quad \beta=1,2,4, \\
\beta^{\prime} & =\frac{k(k+1)}{2} \beta+(k-1), \quad k \geq 1 .
\end{aligned}
$$

This scaling relation is the central result of this paper. Note that $4 \leq \beta^{\prime}<\infty$ can take large integer values and, unlike $\beta=1,2,4$, does not have corresponding random matrix model as yet. Thus, Eq. 3 may be considered as a generalization of the Wigner surmise, that holds good for integer values of $\beta>0$. For $0 \leq \beta \leq 1$, a result that can be considered a special case of the scaling relation Eqs. 3, 4 has been proved for generalized $\beta$ ensembles at the level of the joint probability distribution of eigenvalues 25 . It is also pertinent to point out that similar relation between the higher-order and nearest neighbor spacing distributions had been proposed earlier without rigorous proof ${ }^{26,27}$, though their validity had never been tested on spectra from random matrices or physical systems. One exception is the well-known relation that the next-nearest neighbor $(k=2)$ level spacings of levels from circular orthogonal ensemble are distributed as the nearest neighbor $(k=1)$ spacings of levels from circular symplectic ensemble ${ }^{28}$. In the limit of large matrix dimensions, this is known to be valid for the corresponding Gaussian ensemble as well.

Remarkably, the functional form of $P^{k}(r, \beta)$ is identical to $P\left(r, \beta^{\prime}\right)$ with order of the spacing ratio $k$ and Dyson index $\beta$ dependence entering through the modified parameter $\beta^{\prime}$. In the rest of the paper, numerical evidence from random matrices and from physical systems such as spin chain, quantum billiards and measured nuclear resonances are presented, apart from some well-studied models of quantum chaos like the kicked top and the intermediate map. Further, Eq. 3] suffers from strong finite size effects and we discuss disparate cases that have different rates of convergence to Eq. 4

\section{RESULTS}

\section{A. Random matrix spectra}

First, Eqs. 34 are verified for the spectra computed from random matrix ensembles. The eigenvalues of random matrices (drawn from Gaussian ensembles) of order $N=10^{5}$ are computed for $\beta=1,2$ and 4 . The resulting histograms of higher-order spacing ratios shown in Fig. 1 are averaged over 1000 realizations. The solid curves in this figure represent $P\left(r, \beta^{\prime}\right)$ and its excellent agreement with the histograms points to the validity of Eq. 3. Corresponding averages $\langle r\rangle_{t h}$, as calculated theoretically from Eq. 3, and determined from numerics $\langle r\rangle_{G}$

\begin{tabular}{|c|c|c|c|c|c|}
\hline$\beta$ & $\mathrm{k}$ & $\beta^{\prime}$ & $\langle r\rangle_{t h}$ & $\langle r\rangle_{G}$ & $\langle r\rangle_{C}$ \\
\hline 1 & 2 & 4 & 1.1747 & 1.1757 & 1.1767 \\
& 4 & 8 & 1.0855 & 1.0847 & 1.0860 \\
& & 13 & 1.0521 & 1.0518 & 1.0524 \\
\hline 2 & 3 & 7 & 1.0980 & 1.0976 & 1.0969 \\
& 4 & 23 & 1.0293 & 1.0289 & 1.0291 \\
& 2 & 13 & 1.0521 & 1.0522 & 1.0525 \\
& 3 & 26 & 1.0259 & 1.0258 & 1.0262 \\
& 4 & 43 & 1.0156 & 1.0156 & 1.0158 \\
\hline
\end{tabular}

TABLE I. The average value of $r$, as calculated theoretically from Eq. $3\left(\langle r\rangle_{t h}\right)$ and as determined numerically from data for Gaussian $\left(\langle r\rangle_{G}\right)$ and circular ensembles $\left(\langle r\rangle_{C}\right)$ is shown for different values of $k$ and $\beta^{\prime}$.

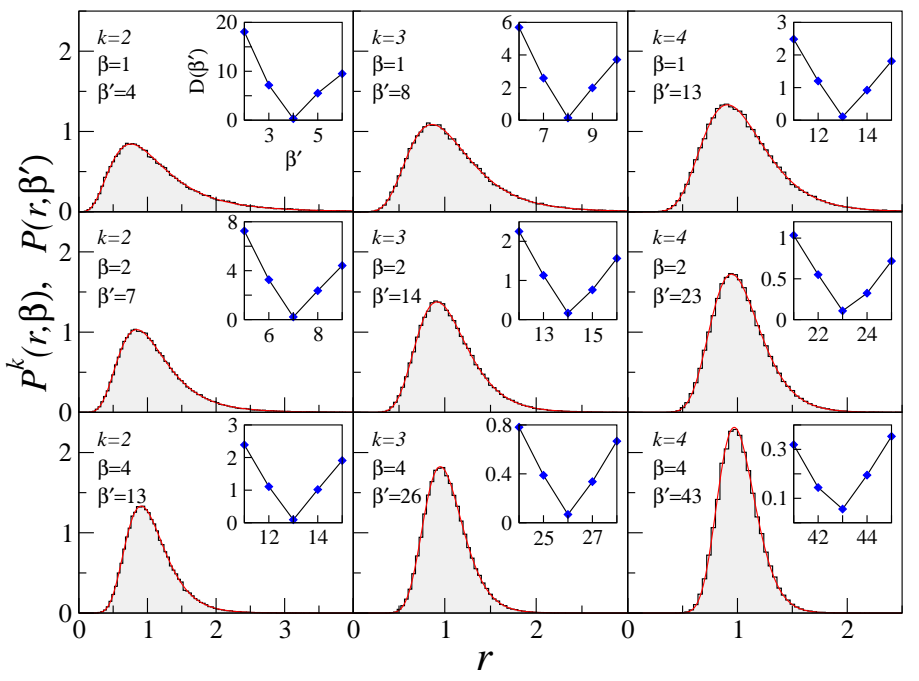

FIG. 1. Distribution of $k$-th order spacing ratios (histograms) for the spectra of random matrices drawn from GOE, GUE and GSE and the distribution $P\left(r, \beta^{\prime}\right)$ (solid line) with $\beta^{\prime}$ given by Eq. 4 (Inset) shows $D$ as a function of $\beta^{\prime}$.

are shown in Table 1] The scaling in Eqs. 3,4 holds good for the circular ensembles of random matrix theory as well. An excellent agreement with the postulated scaling relation is observed (not shown here) for the circular orthogonal, unitary and symplectic ensembles (COE, CUE, and CSE respectively) as well, and the corresponding results for averages $\langle r\rangle_{C}$ are displayed in Table I

Further, to quantitatively check that the value of $\beta^{\prime}$ predicted by Eq. 4 is precisely the one that best fits the histogram $P^{k}(r, \beta)$ obtained from random matrix simula- 
tions, we compute the difference between the cumulative distributions defined as,

$$
D\left(\beta^{\prime}\right)=\sum_{i}\left|I^{k}(r, \beta)-I\left(r_{i}, \beta^{\prime}\right)\right|,
$$

where $I\left(r, \beta^{\prime}\right)$ and $I^{k}(r, \beta)$ are the cumulative distributions corresponding, respectively, to $P\left(r, \beta^{\prime}\right)$ and $P^{k}(r, \beta)$. Then, the value of $\beta^{\prime}$ for which $D\left(\beta^{\prime}\right)$ is minimum is the one that best fits the observed histogram. The insets in Fig. 1 display the quantitative verification of scaling in Eqs. 34. As seen in the insets, the minima of $D\left(\beta^{\prime}\right)$ remarkably coincides with the value of $\beta^{\prime}$ predicted by Eq. 4. As an additional verification, the Kolmogorov-Smirnov test $\underline{29}$ has also been performed for this data, and the $p$-values obtained indicate that the histograms correspond to the predicted distribution in Eq. 3 with a very high probability.

\section{B. Many-body systems (GOE class)}

Figure 2 shows validity of the scaling relation (Eqs. 3.44) for two many-body systems, namely, (i) a onedimensional disordered spin- $1 / 2$ chain, and (ii) for experimentally measured nuclear resonances of the Erbium atom. Both are examples of many-body systems whose nearest-neighbor spectral statistics had been wellestablished as coinciding with that of $\mathrm{GOE}^{30,31}$. The eigenvalues for the spin chain are obtained by diagonalizing the Hamiltonian ${ }^{33}$

$$
\begin{aligned}
H & =\sum_{i=1}^{L} \omega S_{i}^{z}+\epsilon_{d} S_{d}^{z} \\
& +\sum_{i=1}^{L-1}\left[J_{x y}\left(S_{i}^{x} S_{i+1}^{x}+S_{i}^{y} S_{i+1}^{y}\right)+J_{z} S_{i}^{z} S_{i+1}^{z}\right]
\end{aligned}
$$

Here, $L$ is the length of the chain and $S_{i}^{x, y, z}$ are the spin operators in three directions, acting on site $i$. The first term of the Hamiltonian represents a static magnetic field in the $z$-direction, accounting for a Zeeman splitting of strength $\omega$ at all sites, except the defect site $d$ where it is $\epsilon_{d}+\omega$. The second term, by itself is the well-known XXZ Hamiltonian, and couples nearest-neighbor spins in all directions, with $J_{x y}$ (taken here to be 1) being coupling strength along $x$ - and $y$ - directions, and $J_{z}$ (taken as $0.5)$ that along the $z$-direction. For the spectra from the Hamiltonian in Eq. 6, the upper panel of Fig. 2 displays a good agreement between the computed $k$-th spacing ratio distribution and $P\left(r, \beta^{\prime}\right)$ given by Eqs. 3,4 Finite size effects have been discussed for this system in Fig. 5(c), by varying $L$, which changes the Hilbert space dimension. For the distributions shown in Figs. 2(a-d), the length of the spin chain was considered to be $L=14$, the site of the disorder was taken to be at $L / 2$, and the magnitude of the disorder was $\epsilon_{d}=0.5$. A similar excellent agreement can be inferred from the lower panel of Fig. 2 for the

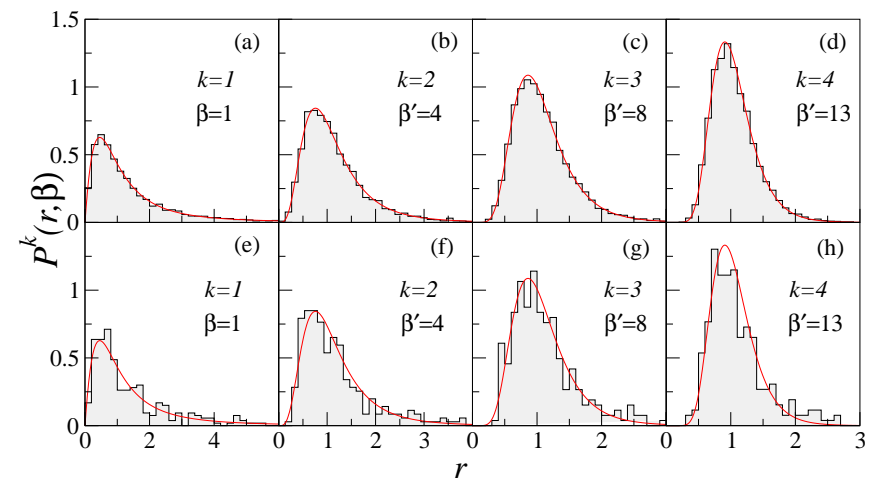

FIG. 2. Distribution of $k$-th spacing ratio for many-body systems of the GOE class $(\beta=1)$. The histograms are for the computed spectra from a disordered spin chain (upper panel) and nuclear resonance of ${ }^{167} \mathrm{Er}$ atom (lower panel). The solid line corresponds to $P\left(r, \beta^{\prime}\right)$ predicted by Eqs. 3.4. with $\beta^{\prime}=1,4,8$ and 13 for $k=1$ to 4 .

experimentally measured data for neutron resonances of the Erbium atom ${ }^{32}$. Even with only about 200 measured resonances, a good agreement with the theoretical form of $P^{k}\left(r, \beta^{\prime}\right)$ is observed for $k=1$ to 4 .

\section{Disordered spin chain and quantum chaotic system (GUE class)}

The validity of Eqs. 3, 4 for two physical systems belonging to GUE symmetry class is discussed. They are, (i) a one-dimensional disordered spin- $1 / 2$ system $^{34}$, and (ii) a quantum billiard without time reversal symmetry 35 . They are not invariant under time reversal symmetry and hence belong to the GUE class. The Hamiltonian for the disordered spin chain is

$$
H=\sum_{i=1}^{L}\left[J_{1}\left(\mathbf{S}_{i} \cdot \mathbf{S}_{i+1}\right)+h_{i} S_{i}^{z}+J_{2}\left(\mathbf{S}_{i} \cdot\left(\mathbf{S}_{i+1} \times \mathbf{S}_{i+2}\right)\right],\right.
$$

in which $J_{1}$ and $J_{2}$ represent strength of coupling between sites. The first term (by itself, the Heisenberg spin chain) corresponds to nearest neighbor couplings in all directions, with $J_{1}$ giving the strength of the coupling. The second term introduces a Gaussian distributed, random magnetic field of mean 0 and strength $h_{i}$ in the $z$-direction. The third term breaks time reversal symmetry by introducing a three-spin interaction with the nearest as well as the next-nearest neighbor couplings with strength $J_{2}$. The parameters used to obtain data for Figs. 3(a-d) are $L=12, h / J_{1}=1$ and $J_{2} / J_{1}=1$, with open boundary conditions. The computed spacing ratio distribution $P^{k}\left(r_{i}, \beta=2\right)$ shown in the upper panel of Fig. 3 for $k=2,3,4$ is consistent with $P\left(r, \beta^{\prime}\right)$.

An experimentally realized quantum chaotic billiard 36 has been simulated here, which is a microwave cavity (of size $\sim 17$ inches) placed in a static magnetic field, in 


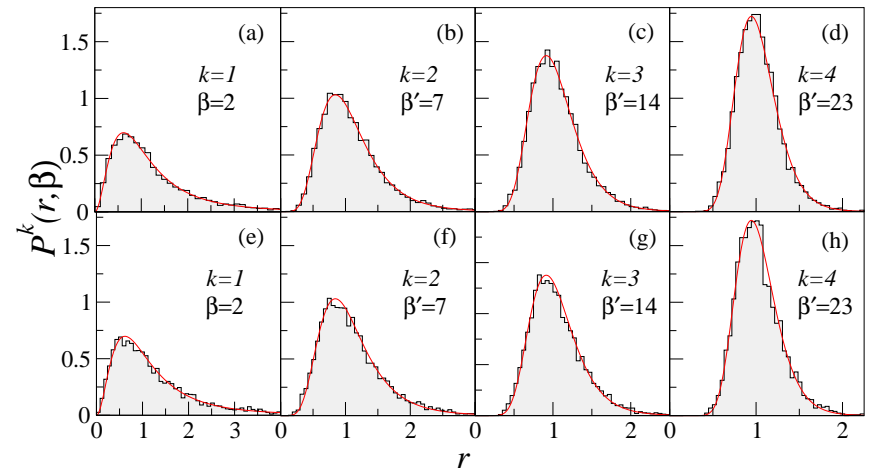

FIG. 3. Distribution of $k$-th spacing ratio for physical systems of the GUE class $(\beta=2)$. Histogram is for a spin chain with a three-spin interaction (upper panel), and chaotic billiards with a magnetized ferrite strip (lower panel). The solid line represents the predicted $P\left(r, \beta^{\prime}\right)$, with $\beta^{\prime}=2,7,14$ and 23 for $k=1$ to 4 .

which time reversal symmetry is broken due to the presence of a magnetizd ferrite strip placed on one of the walls. The simulation uses exactly the same configuration and parameters described in Ref ${ }^{36}$. The eigenvalues were computed by solving the Helmholtz equation with suitable boundary conditions, and its fluctuations coincide with GUE statistics. As seen in lower panel of Fig. 3. the distribution of $k$-th spacing ratios provides another instance of the validity of the scaling relation in Eqs. 34 4 for GUE systems with $\beta=2$.

\section{Spectra of Floquet systems}

The validity of Eq. 3,4 has also been tested on timedependent (driven) systems whose time-evolution operators (Floquet matrices) have unimodular eigenvalues. Their fluctuation properties are modeled by those of circular random matrix ensembles. One relevant model is the quantum kicked top whose classical limit is chaotic 37 . As this system is periodically kicked, the quantum version can be studied in terms of the unitary time evolution operator

$$
\widehat{U}=\exp \left(-i q J_{z}^{2} / 2\right) \exp \left(-i p J_{y}\right),
$$

where $q=10$ is the kick strength that acts as chaos parameter and $p=1.7$. The action of this operator on a particle of angular momentum $\mathbf{J}$, taken to be 200 here, is a precession about the $y$-axis, followed by state-dependent rotation about the $z$-axis as a consequence of periodic kicking. The eigenvalues of $\widehat{U}$ are computed by diagonalizing this operator and its fluctuations are known to be consistent with COE statistics 37 . Figure 4 (upper panel) shows the $k$-th spacing ratio distribution for this system which, as anticipated by Eqs. 3, 4, follows $P\left(r, \beta^{\prime}\right)$ with $\beta=1$.

As another instance of CUE class $(\beta=2)$, a unitary operator corresponding to the so-called intermedi-

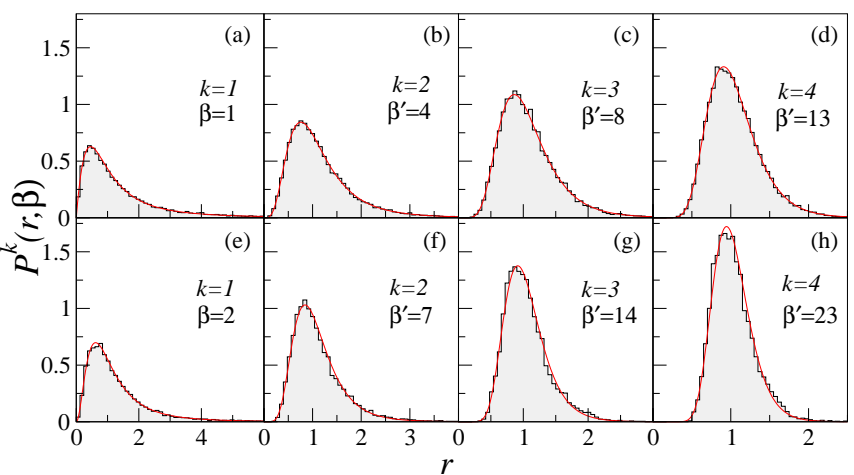

FIG. 4. The distribution of the $k$-th spacing ratios, for $k=1$, 2, 3, 4 is shown for Floquet systems; (upper panel) the kicked top, belonging to the COE class, and (lower panel) the intermediate map, belonging to the CUE class. The histograms are obtained from computed eigenvalues of these systems, and the solid line represents $P\left(r, \beta^{\prime}\right)$, with $\beta^{\prime}=1,4,8,13$ for COE and $\beta^{\prime}=2,7,14,23$ for CUE.

ate map is considered. The quantum version of this map has been investigated previously in the context of multifractal eigenstates, and in a specified range, has spectral fluctuations similar to CUE matrices 38 . The unitary operator can be written in terms of an $N \times N$ matrix as

$$
U_{a b}=\frac{\exp \left(-i \phi_{a}\right)}{N} \frac{1-\exp [i 2 \pi \gamma N]}{1-\exp [i 2 \pi(a-b+\gamma N) / N]},
$$

with Hilbert space dimension $N=12000$. Here, $\phi_{a}$ is a random variable uniformly distributed between $[0,2 \pi]$, and for any irrational $\gamma$ the spectral statistics is of the CUE type. The computed distribution of $k$-th spacing ratios for this system, shown in Fig. 4 (lower panel), agrees well with Eqs. 3, 4

\section{FINITE SIZE EFFECTS}

The scaling relation in Eqs. 34 suffers from finite size effects with different systems converging to the scaling relation at different rates, especially if $k>>1$. In the spectra of physical systems as well as in the random matrices of the Gaussian and circular ensembles, it was observed in practice that for higher order spacing ratios, say $k>5$, the value of $\beta^{\prime}$ obtained by fitting $P\left(r, \beta^{\prime}\right)$ to the empirical distribution did not quite agree with that predicted by Eq. 4. It is seen that the convergence to the predicted $\beta^{\prime}$ is strongly pronounced as the order $N$ of the random matrix increases. This is illustrated in Fig 5 for two distinct values of $k$. In one case, for $k=9$ and based on Eq. 4, the expected value of $\beta^{\prime}=53$. Fig. [5)(a) shows a clear convergence to this predicted value as the order $N$ of the random matrix increases. For $k=20$, Eq. 4 predicts $\beta^{\prime}$ to be 229 . However, as seen in Fig. 5(b), the convergence to the predicted value of $\beta^{\prime}$ is rather slow, and up to $N=40000$ for which spectra was computed it 


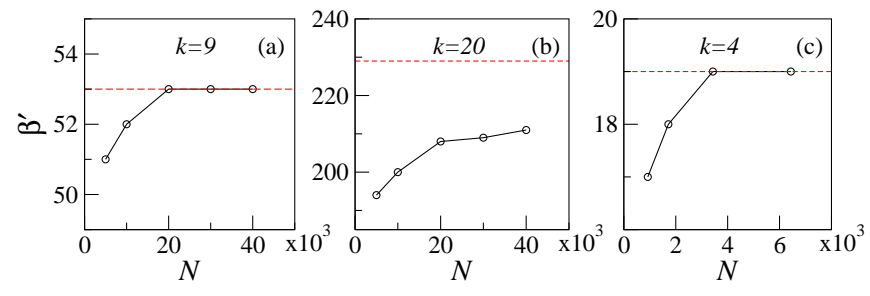

FIG. 5. Variation of $\beta^{\prime}$ as a function of matrix dimension $N$, for random matrices of the GOE class, for (a) $k=9$ and (b) $k=20$. For $k=9, \beta^{\prime}$ converges to the predicted value $\left(\beta^{\prime}=53\right)$ as $N$ increases, while for $k=20$, a steady increase of $\beta^{\prime}$ towards the predicted value of $\beta^{\prime}=229$ is observed. (c) Variation of $\beta^{\prime}$ as a function of matrix dimension $N$ for the GOE spin chain (Eq. 6). In this case, as $N$ increases, $\beta^{\prime}$ converges to 19 , the predicted value.

had not converged at all. Fig. 5(c) shows the same effect for the GOE spin chain (Eq. 6) for $k=4$ as a function of the size of Hilbert space for the system. In this case, the dimension $N$ of the Hamiltonian matrix increases upon increasing the length $L$ of the spin chain. As Fig. 5(c) reveals convergence is achieved for $N \approx 40000$.

In physical systems, it is well-known that purely quantum effects such as tunneling and localization lead to deviations from random matrix averages.Hence, in physical systems, the deviations from scaling could arise due to both physical effects that are not accounted for by RMT-type universality and also the finite size effects. By studying these deviations in physical systems from expectations based on Eqs. 3,4] and comparing it with random matrices of identical dimensions in which the deviations are purely due to finite size effects, it might be possible to distinguish whether the deviations occur due to finite size effects or system-dependent causes. The distribution of higher-order ratios may then be useful to differentiate between and understand the effects of random and system-dependent fluctuations in any physical system.

\section{CONCLUSIONS}

In summary, a generalized form of the Wigner surmise has been proposed to obtain a form for the distribution of non-overlapping spacing ratios of higher-orders, in which the Dyson index $\beta \geq 4$ for the Gaussian and circular ensembles of random matrix theory. An elegant scaling relation is observed, relating the order $k$ of the spacing ratio and the Dyson index $\beta$, to another constant $\beta^{\prime}$. Effectively, for large random matrix sizes $N>>1$, the form of distribution of higher-order spacing ratios $P^{k}\left(r, \beta^{\prime}\right)$ may be obtained for any arbitrary $k$, given the class of random matrices being considered. For sufficiently small $N$ and large $k$, finite size effects do tend to induce deviations from the proposed scaling relation. It has been shown that convergence to scaling relation can be restored by increasing the dimension of the random matrix. In some cases, convergence to the predicted value was observed to be faster when the $k$-th order ratios considered were calculated in a completely non-overlapping manner, i.e. in Eq. 2, $i$ increases in steps of $k$. Here, the numerator of the $i^{t h}$ ratio, would be the denominator of the $(i+1)^{t h}$ ratio. The scaling relation Eq. 4 remains unchanged when the distribution of these ratios are considered as well.

This scaling is shown to be valid for several different physical systems, namely, spin chains belonging to GOE and GUE class, measured resonances of Er atom, chaotic billiards, Floquet systems, all of whose eigenvalue fluctuations properties are well described by an appropriate ensemble of random matrix theory. It must be noted that higher-order spacing ratios are far easier to compute compared to the spacing distribution beyond the nearest neighbor. The results proposed here are of inherent interest in random matrix theory and provide large number of additional statistic to easily test the fluctuation properties of physical systems for putative RMTtype behavior. The rigorous numerical results proposed in this work should lead to attempts to obtain analytical justification for them.

Acknowledgements: The authors acknowledge Sanku Paul for providing data for the levels of the intermediate map. UTB acknowledges the funding received from Department of Science and Technology, India under the scheme Science and Engineering Research Board (SERB) National Post Doctoral Fellowship (NPDF) file number $\mathrm{PDF} / 2015 / 00050$.
* E-mail: harshini.t@gmail.com

$\dagger$ E-mail: udaybhosale0786@gmail.com

\#-mail: santh@iiserpune.ac.in

1 H. Hasegawa, and Y. Sakamoto, Progress of Theoretical Physics Supplement, 139, 112 (2000); S. M. Nishigaki, Phys. Rev. E, 59, 2853 (1999).

2 S. D. Geraedts, R. Nandkishore and N. Regnault, Phys. Rev. B, 93, 174202 (2016).

3 L. Reichl, The Transition to Chaos: Conservative Classi- cal Systems and Quantum Manifestations, Springer Science and Business Media (2013).

4 O. Bohigas, M. J. Giannoni and C. Schmidt, Phys. Rev. Lett. 52, 1 (1984).

5 M. L. Mehta, Random Matrices (Academic Press, New York, 2004)

6 Usually, spacings are taken in units of mean level spacing for comparing with Wigner distribution. E.P. Wigner, Mathematical Proceedings of the Cambridge Philosophical 
Society, 47, 790 (1951).

7 J. X. Zhong, U. Grimm, R. A. Roemer and M. Schreiber, Phys. Rev. Lett. 80, 3996 (1998); F. Siringo, and G. Piccitto, J Phys. A: Mathematical and General, 31, 5981 (1998).

8 R. Modak, S. Mukerjee, and S. Ramaswamy, Phys. Rev. B 90, 075152 (2014); M. Serbyn and J.E. Moore, Phys. Rev. B, 93, 041424 (2016).

9 H. J. Stöckmann, Quantum Chaos: An Introduction, Cambridge U.P. (2000).

10 K. M. Atkins and G. S. Ezra, Phys. Rev. E, 51, 1822 (1995).

11 H. Friedrich and H. Wintgen, Phys. Rep. 183, 37 (1989).

12 H. A. Weidenmüller and G. E. Mitchell, Rev. Mod. Phys. 81, 539 (2009); F. M. Izrailev, Phys. Rep. 196, 299 (1990); H. J. Stöckmann and J. Stein, Phys. Rev. Lett. 64, 2215 (1990); D. Delande, and J. C. Gay, Phys. Rev. Lett. 57, 2006 (1986); V. K. B. Kota, Embedded random matrix ensembles in quantum physics (Vol. 884), Heidelberg: Springer (2014).

13 M. Serbyn and J. E. Moore, Phys. Rev. B 93, 041424(R) (2016); J. A. Kjäll, Phys. Rev. B 97, 035163 (2018); E. Canovi, New J. Phys. 14, 095020 (2012); R. Vosk, D. A. Huse and E. Altman, Phys. Rev. X 5, 031032 (2015); V. K. Varma et. al., J. Stat. Mech. 053101 (2017); P. Sierant, D. Delande and J. Zakrzewski, Phys. Rev. A 95, 021601(R) (2017).

14 V. Oganesyan, D. A. Huse, Phys. Rev. B 75, 155111 (2007).

15 C. Kollath, G. Roux, G. Biroli and A. M. Läuchli, J. Stat. Mech. P08011 (2010); M. Collura, H. Aufderheide, G. Roux and D. Karevski, Phys. Rev. A 86, 013615 (2012).

16 Y. Y. Atas, E. Bogomolny, O. Giraud, and G. Roux, Phys. Rev. Lett. 110, 084101 (2013).

17 Y. Y. Atas et. al., J. Phys. A : Math. Theor. 46, 355204 (2013).

18 N. D. Chavda, H. N. Deota and V. K. B. Kota, Phys. Lett. A, 378, 3012 (2014); V. K. B. Kota and N. D. Chavda, International Journal of Modern Physics E, 27, 1830001 (2018); S. Harshini Tekur, Santosh Kumar and M. S. Santhanam, Phys. Rev. E, 97, 062212 (2018).

19 There is no unique definition of higher spacing ratio and here we adopt the convention that non-overlapping ratio implies no shared eigenvalue spacing between the numerator and denominator in Eq. 2. See Ref. ${ }^{17}$ for one result related to overlapping higher-order spacing ratio distribution.
20 M. Brack and R. K. Bhaduri, Semiclassical Physics, Westview Press (2003); M.C. Gutzwiller, Chaos in Classical and Quantum Physics, Springer New York (1990).

${ }^{21}$ K. Agarwal, E. Altman, E. Demler, S. Gopalakrishnan, D. A. Huse, and M. Knap, Ann. Phys. (Berlin) 529, 1600326 (2017).

22 T. Guhr, A. Muller-Groeling and H. A. Weidenmüller, Phys. Rep. 299, 189 (1998).

23 V. E. Kravtsov, I. M. Khaymovich, E. Cuevas, M. Amini, New J. Phys. 17, 122002 (2015).

24 P. J. Forrester and E. M. Rains, Probability theory and related fields, 130, 518 (2004).

25 P. J. Forrester, Comm. math. phys. 285, 653 (2009).

26 P. B. Kahn and C. E. Porter, Nucl. Phys. 48, 385 (1963).

27 A. Y. Abul-Magd, and M. H. Simbel, Phys. Rev. E, 60, 5371 (1999).

28 M. L. Mehta and F. J. Dyson, Journal of Mathematical Physics, 4 (1963).

29 D. C. Boes, F. A. Graybill and A. M. Mood, Introduction to the theory of statistics, 3rd ed., New York: McGraw-Hill (1974).

30 L. F. Santos, J. Phys. A: Mathematical and General, 37, 4723 (2004); K. Kudo and K. Nakamura, Phys. Rev. B, 71, 144427 (2005); D. J. Luitz, N. Laflorencie and F. Alet, Phys. Rev. B, 91, 081103 (2015).

31 O. Bohigas and H. A. Weidenmuller, Annual Review of Nuclear and Particle Science, 38, 421 (1988); V. Zelevinsky and A. Volya, Physica Scripta, 2006(T125), 147 (2006).

${ }^{32}$ H. I. Liou, H. S. Camarda, S. Wynchank, M. Slagowitz, G. Hacken, F. Rahn, and J. Rainwater, Phys. Rev. C 5, 974 (1972).

33 A. Gubin and L. F. Santos, Am. J. Phys. 80, 246 (2012).

34 Y. Avishai, J. Richert, and R. Berkovits, Phys. Rev. B 66, 052416 (2002).

35 H. Schanze, H. -J. Stöckmann, M. Martínez-Mares, and C. H. Lewenkopf, Phys. Rev. E 71, 016223 (2005); B. Dietz, T. Friedrich, H. L. Harney, M. Miski-Oglu, A. Richter, F. Schäfer, and H. A. Weidenmüller, Phys. Rev. Lett. 98, 074103 (2007).

36 P. So, S. M. Anlage, E. Ott, and R. N. Oerter, Phys. Rev. Lett. 74, 2662 (1995).

37 F. Haake, M. Kuś, and R. Scharf, Zeitschrift fr Physik B Condensed Matter 65, 381 (1987); S. Chaudhury, A. Smith, B. E. Anderson, S. Ghose, and P. S. Jessen, Nature, 461, 768 (2009).

38 R. Dubertrand, I. Garcia-Mata, B. Georgeot, O. Giraud, G. Lemarie, and J. Martin, Phys. Rev. E 92, 032914 (2015). 